\title{
Self-shielded electron linear accelerators designed for radiation technologies
}

\author{
V. M. Belugin and N.E. Rozanov \\ Moscow Radiotechnical Institute of Russian Academy of Sciences, 132 Warshavskoe shosse, Moscow 113519 \\ V. M. Pirozhenko* \\ Carlsbad, California 92009, USA
}

(Received 24 February 2009; published 10 September 2009)

\begin{abstract}
This paper describes self-shielded high-intensity electron linear accelerators designed for radiation technologies. The specific property of the accelerators is that they do not apply an external magnetic field; acceleration and focusing of electron beams are performed by radio-frequency fields in the accelerating structures. The main characteristics of the accelerators are high current and beam power, but also reliable operation and a long service life. To obtain these characteristics, a number of problems have been solved, including a particular optimization of the accelerator components and the application of a variety of specific means. The paper describes features of the electron beam dynamics, accelerating structure, and radio-frequency power supply. Several compact self-shielded accelerators for radiation sterilization and x-ray cargo inspection have been created. The introduced methods made it possible to obtain a high intensity of the electron beam and good performance of the accelerators.
\end{abstract}

DOI: 10.1103/PhysRevSTAB.12.090101

PACS numbers: 29.20.Ej, 29.27.Bd, 41.20.Jb

\section{INTRODUCTION}

Currently, there is a demand for electron linear accelerators with high beam power and local radiation shielding. They are designed for the radiation sterilization of medical devices and materials, $x$-ray inspection of containers, cars and trucks, etc. The application of self-shielded accelerators makes it possible to avoid construction of special buildings, as well as to promote and make less expensive the creation of radiation technology systems. In particular, it provides the possibility of developing radiation sterilization systems that can be used in clinics and small facilities.

Accelerators of this type should meet several specific requirements. They should have an energy of accelerated electrons in the range of 3 to $10 \mathrm{MeV}$ and a beam power of no less than $1 \mathrm{~kW}$. The accelerators should have small dimensions and a high acceleration rate in order to minimize the dimensions and weight of the radiation shielding. Usually the shielding contains blocks made from cast iron. Therefore, the accelerators should not use any devices with a magnetic field. Finally, the accelerators designed for radiation technologies should have good operation reliability and a long service life.

A specific property of compact linear accelerators is that they do not use magnetic devices for electron beam focusing. Acceleration and focusing of the beam are performed by rf fields in the accelerating structure. The first experimental accelerator of this type was described in [1]. The

\footnotetext{
*vitaly@pirozhenko.com
}

maximum peak beam current of $130 \mathrm{~mA}$ was obtained in this accelerator.

The development of self-shielded electron linear accelerators has been performed for many years in the Moscow Radiotechnical Institute of Russian Academy of Sciences. Several accelerators for radiation sterilization and $\mathrm{x}$-ray cargo inspection have been created. References [2,3] contain brief information on the accelerators that were developed. The maximum peak beam current of $270 \mathrm{~mA}$ was achieved. Although many efforts on the development of accelerators of this type have been made [4-8], until now this magnitude of beam current has not been exceeded.

The development of self-shielded accelerators with high electron beam current and power has required essential improvements of the accelerators and their components. This paper contains a detailed description of the accelerators, problems in their development, and solutions found.

\section{SCHEME AND CHARACTERISTICS OF THE ACCELERATOR}

\section{A. Scheme of the self-shielded accelerator}

A typical example of a radiation system with a selfshielded electron accelerator is a sterilization system of the TOROS type. Figure 1 shows the design of the system.

The electron accelerator includes the electron injector, accelerating resonator, $r f$ generator, waveguide, and pulse generator. The system also includes the electron beam scanner, the transport mechanism with irradiated objects, the radiation shielding, and auxiliary devices. The accel- 


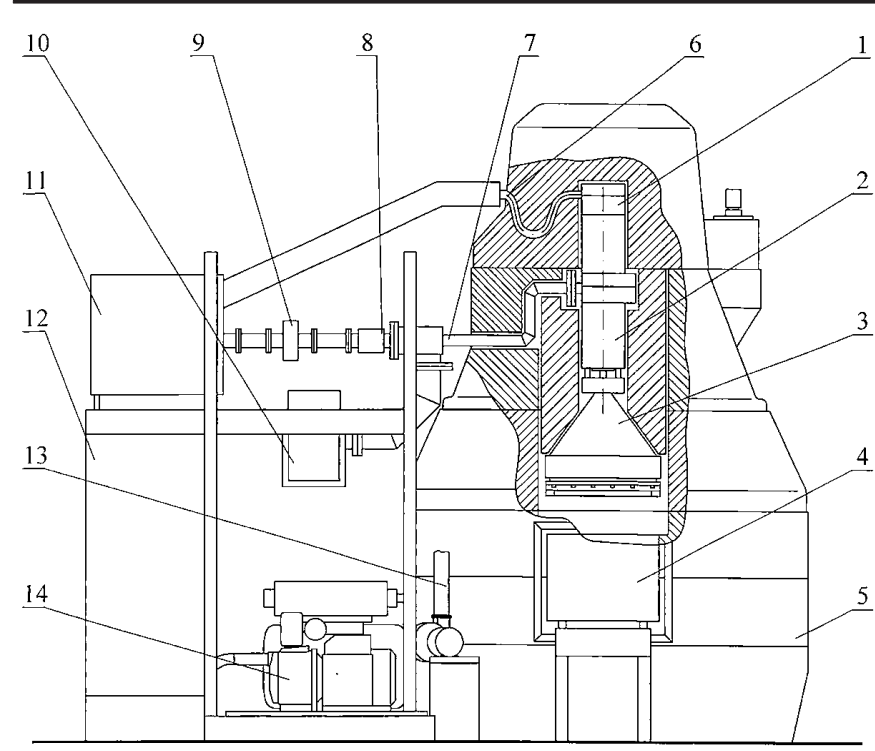

FIG. 1. Design of a sterilization system of the TOROS type: 1-electron injector; 2-accelerating resonator; 3-beam scanner; 4-irradiated object; 5-radiation shielding; 6-highvoltage cable; 7-waveguide; 8-ceramic window; 9-ferrite circulator; 10-vacuum pump; 11-rf generator; 12 - pulse generator; 13-block for ozone ventilation; 14-water cooling block.

erated electron beam travels down and is scanned in the plane perpendicular to the direction of the moving objects.

The accelerating resonator is fed by a rf generator built around an MI-456A magnetron. The magnetron is located between the poles of a permanent magnet and is fed by the pulse generator. The waveguide contains the ferrite circulator, a measuring section with directional couplers, a ceramic window, and a vacuumed section, which is located in the labyrinth of the radiation shielding.

Vacuum pumping of the resonator is carried out through the waveguide. The injector is pumped out by the separate vacuum pump. The water cooling block is designed to cool the resonator and magnetron and control their temperatures.

The radiation shielding ensures reliable protection of the operating personnel from scattered x-rays. The shielding consists of blocks manufactured from cast iron [9]. The shielding has the complicated shape and variable thickness that have been calculated and optimized with regard to the intensities, energies, and distribution of the scattered $\mathrm{x}$ rays. $\mathrm{X}$ rays are emitted from the irradiation area, from the electron beam scanner, and from the accelerating resonator, wherein the beam loses some particles. The x-ray emission rate from the irradiation area is much more than from the resonator and beam scanner. So the shielding thickness is defined mainly by the $\mathrm{x}$-ray emission rate from the irradiation area. Usually measured values of radiation levels at $1 \mathrm{~m}$ distance from the radiation shielding are no more than $3 \mu \mathrm{Sv} / \mathrm{hr}$.

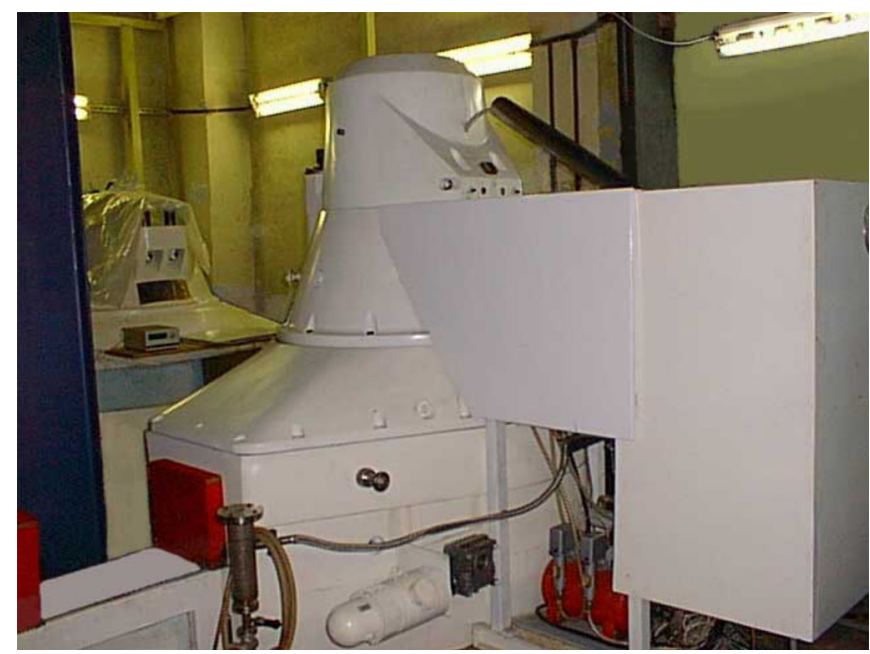

FIG. 2. (Color) Photo of TOROS system.

Figure 2 shows a photograph of the sterilization system. The system includes a linear transport mechanism for the boxes with medical products. The electron accelerator operates continuously, and the required radiation protection is assured at every position of the transport mechanism. For this purpose, the transport mechanism contains three shielding blocks and two ports between them.

The $\mathrm{x}$-ray inspection system of the POLISCAN-3 type also contains the self-shielded electron accelerator [10]. The accelerator has a similar scheme, but there is a conversion target at the accelerator end that converts the electron beam energy to $\mathrm{x}$ rays. The accelerator and target are placed inside the local radiation shielding with the collimator forming a narrow, fan-shaped $\mathrm{x}$ ray that irradiates inspected objects.

\section{B. Scheme of the accelerating structure}

Use of local radiation shielding places a constraint on the design of an accelerating structure. Standing-wave (SW) structures with the $\pi / 2$ mode of operation are used in self-shielded compact electron accelerators. They have high efficiency, high acceleration rate, and good stability of rf fields in cavities. They can provide the forming and acceleration of electron bunches without use of a focusing solenoid.

A few types of SW accelerating structures for $\mathrm{rf}$ linear accelerators have been developed [11-13]. The on-axis coupled structure is best suited for compact electron accelerators since it has a smaller diameter than other structures. Figure 3 shows the scheme of the accelerating resonator and electron injector.

The resonator contains a biperiodic chain of cavities. The accelerating cavities have an optimized shape to reduce rf power losses and electric field gradients. The cavity-to-cavity coupling is provided by the magnetic field via off-center slots. The phase shift between adjacent cavities is $\pi / 2$ and between accelerating cavities is $\pi$. 


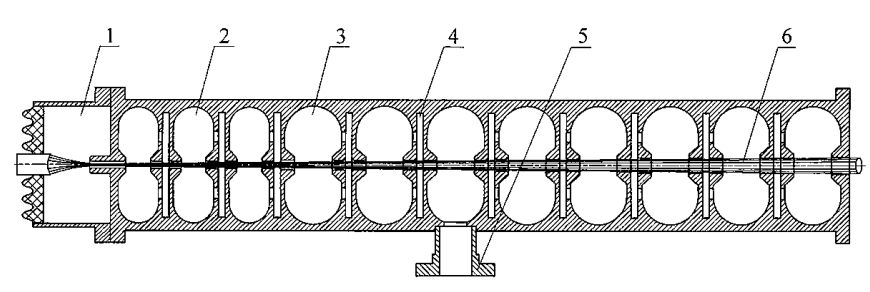

FIG. 3. Scheme of accelerating resonator and electron injector: 1-electron injector; 2-cavity of bunching section; 3-cavity of accelerating section; 4-coupling cavity; 5-waveguide; 6electron beam.

The resonator includes the bunching and the accelerating sections. The bunching section contains several cavities with specific dimensions and field amplitudes that execute the forming and preliminary acceleration of electron bunches. The accelerating section contains cavities with equal dimensions and field amplitudes that execute the main acceleration of electrons to the required energy.

The electron injector contains a cathode, a focusing electrode, and a high-voltage ceramic insulator. The feeding waveguide is connected to the middle cavity through a rectangular-shaped window. The window dimensions are adjusted to match the waveguide and resonator.

\section{Characteristics of accelerators}

The main characteristics of the electron accelerators used in the TOROS sterilization system and the POLISCAN-3 inspection system are listed in Table I.

In the accelerators of the TOROS systems, a maximum peak current of the electron beam of $270 \mathrm{~mA}$ has been obtained. However, reliable long-term operation with stable characteristics of the accelerated electron beam is usually performed at a peak current of $250 \mathrm{~mA}$.

The accelerators of the POLISCAN-3 system have higher electron energy and greater pulse duration. These parameters are needed to obtain the required signals from radiation detectors and to provide good penetration of the inspection system.

\section{BEAM DYNAMICS}

\section{A. Problems of acceleration of high-intensity beams}

Calculations of the electron beam dynamics in the accelerators have been performed by the DINA program complex, which uses the method of "macroparticles" and includes the following codes [14]: (i) DINA-ROZ-the 2.5D code for the description of the formation and acceleration of electron beams in a steady-state, periodic-intime regime; (ii) DINA-TIME- the $2.5 \mathrm{D}$ code for calculations of beam evolution in transition stages, without an assumption of the process periodicity in time; (iii) DINA3D-the 3D code for computer simulation of electron beam dynamics in a steady-state, periodic-in-time regime.

The process of concurrent bunching, focusing, and acceleration of the electron beam is complicated. As is known, it is impossible to ensure long-term combined longitudinal and transversal stabilities of a beam in a rf accelerator without application of an external magnetic field. So it is necessary that it operates in a transition regime minimizing instabilities by system optimization.

In a high-intensity accelerator, optimization of beam dynamics has specific features. As the calculations show, it is possible to obtain a sufficiently large coefficient of capture of the injected beam to acceleration. However, operating experience shows that other characteristics are also of importance for the reliable and long-term operation of accelerators of this type.

The electrons injected into the accelerator may be separated into four categories: (i) Some electrons are accelerated and emerge from the accelerator; this is the useful part of the beam. (ii) Some electrons stop, drift in all directions, and are incident on the walls of cavities, having low energies. (iii) Some electrons are accelerated, gradually defocused, and are stranded on walls of the accelerator

TABLE I. Main characteristics of accelerators.

\begin{tabular}{lcc}
\hline \hline System & TOROS & POLISCAN-3 \\
\hline Electron energy $(\mathrm{MeV})$ & 5 & 7.3 \\
Peak current of accelerated beam $(\mathrm{mA})$ & 250 & 150 \\
Pulse duration $(\mu \mathrm{s})$ & 6 & 12 \\
Pulse repetition rate $(\mathrm{pps})$ & 300 & 100 \\
Average power of accelerated beam $(\mathrm{kW})$ & 1.8 & 1 \\
Electron injector voltage $(\mathrm{kV})$ & 40 & 40 \\
Length of accelerator $(\mathrm{m})$ & 0.7 & 1.0 \\
Operating frequency $(\mathrm{MHz})$ & 2797 & 2797 \\
Peak power of magnetron $(\mathrm{MW})$ & 2.5 & 2.5 \\
Maximum average power of magnetron $(\mathrm{kW})$ & 4.5 & 4.5 \\
Thickness of radiation shielding (m) & $0.22-0.6$ & $0.25-0.45$ \\
Attenuation of x rays by shielding & $10^{4}-5 \times 10^{7}$ & $10^{4}-10^{5}$ \\
Overall dimensions of radiation shielding $(\mathrm{m})$ & $2.5 \times 1.65 \times 2.6$ & $1.1 \times 1.1 \times 2.35$ \\
\hline \hline
\end{tabular}


channel. They expend rf power, heat the walls, and produce $\mathrm{x}$-rays. (iv) Some electrons reenter the injector, and some bombard the cathode. They may cause overheating and premature failure of the cathode.

The main task of optimization of beam dynamics in the high-intensity accelerator is to decrease the power of the third and fourth parts of the electron beam.

\section{B. Features of beam dynamics 1. Optimization of the bunching section}

High-intensity accelerators with beam focusing by $\mathrm{rf}$ field use extended bunching sections containing several cavities. The electron beam is bunched adiabatically and coincidentally at the beginning of the acceleration. This gives the possibility to bunch a high-intensity beam into compact bunches since the effect of the forces of the beam space charge is greatly moderated at high energies of electrons.

In order to decrease the power of useless parts of the beam, it is profitable to increase the number of cavities in the bunching section and also to reduce field intensities in the initial cavities. But these measures cause an increase of the accelerator length. Therefore, these parameters are chosen as a trade-off.

\section{Expanded aperture of the accelerator}

Of fundamental importance is the selection of the phases of the electrons passing through the cavities. In the major part of the accelerator, the electrons are located on the focusing slope of the rf field. The values of the phases are modest in order to provide a high acceleration rate. Since the focusing forces depend on time, various parts of the beam experience various focusing forces. As the calculations show, the electron beam has a peculiar distribution: it has a high-density core with a very small radius and a lowdensity halo with a large radius [15]. The halo gradually expands as the electrons move along the accelerator.

It was found useful to make the accelerator channel with an increasing radius of the aperture (see Fig. 3). The radius is small at the beginning of the accelerator and increases along the accelerator. It may be increased gradually or by steps. The small aperture at the beginning of the accelerator gives the possibility to stop some extreme electrons of the halo before they gain high energy and consume rf power. The large aperture at the accelerator end makes it possible to pass all electrons with high energy to the accelerator output. It is also useful to place a long tube with a small internal radius between the electron injector and the first cavity. It acts as an aperture filter, decreasing the number of electrons that may reenter the injector and bombard the cathode.

\section{Optimal parameters of the injected beam}

The required parameters of the injected beam are achieved by careful optimization of the shape and dimen- sions of the injector electrodes. The spherically shaped cathode has an $8.4 \mathrm{~mm}$ diameter and good distribution of the emitted beam current. The beam injected into the resonator has a reasonably large diameter, in order that Coulomb forces would not be too large. The beam envelope at the resonator input is not converging or diverging, that is, the resonator input is located at the beam crossover.

In some cases, special requirements may be imposed upon the beam parameters. Particularly in the inspection system, the output electron beam should have reasonably uniform distribution in the cross section. If a thin highdensity core exists on the axis, it may burn through the conversion target. To ensure that the output electron beam has the required radius and good distribution, the beam should have a specific phase portrait. In particular, electrons should have the azimuth components of the velocity. The beam takes the required phase portrait in the injector, which has a specific design of the cathode [16]. During the acceleration in the resonator, this beam retains sufficiently good distribution in the cross section.

\section{Results of beam dynamics optimization}

The described methods were introduced in the accelerators and gave significant improvement of some parameters. Table II shows the change of some parameters of the TOROS accelerator after beam dynamics optimization.

As a result of beam dynamics optimization, the power of stranded electrons decreased significantly. This essentially increased accelerator efficiency. In addition, the power of the scattered radiation from the accelerator became much less. The majority of stranded electrons sits in the first cavity but has small energy. The electrons stranded in the last cavities have small currents but much greater energies. For this reason, the major portion of the radiation is emitted from the accelerator end.

An important feature of the adiabatic beam buncher is that the capture coefficient depends only slightly on the beam current. Therefore, the output beam current is almost directly proportional to the injected one. A decrease of the beam current causes an increase of the rf field intensity and the output energy. Figure 4 shows the calculated and measured characteristics of beam loading in the TOROS accelerator.

The electron energy was measured using transmission of accelerated electrons through an aluminum plate with a

TABLE II. Improvement of parameters of the TOROS accelerator.

\begin{tabular}{lcc}
\hline \hline Variant & Initial & Optimized \\
\hline Average power of stranded electrons $(\mathrm{kW})$ & 0.6 & 0.1 \\
Efficiency of accelerating structure $(\%)$ & 45 & 57 \\
Power of electrons bombarding cathode $(\mathrm{W})$ & 20 & 6 \\
\hline \hline
\end{tabular}




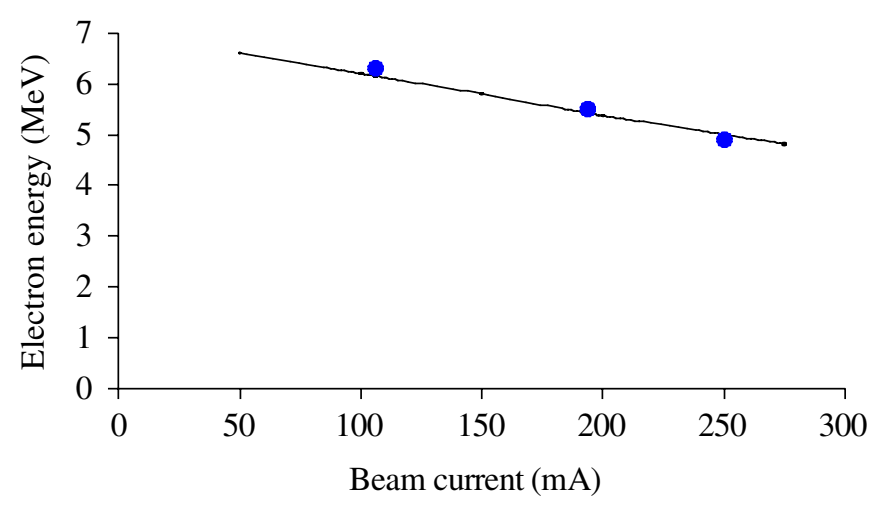

FIG. 4. (Color) Beam loading characteristics of the TOROS accelerator: line_calculations; points-measurements.

$6 \mathrm{~mm}$ thickness. This method gives a value of energy close to the average energy of the electron beam spectrum.

\section{ACCELERATING STRUCTURE}

\section{A. Characteristics of the accelerating structure}

The calculations and optimization of cavities were performed by the 2D code, applying a finite-element method with irregular rectangular net. The calculations of the structure with coupling slots between the cavities were performed applying the 3D code. The adjustment and experimental study of the structure have shown that the codes ensure good accuracy.

The main requirements for the accelerating structure include obtaining a large value of effective shunt impedance, sufficient coupling factor between the cavities, and allowable surface electric fields in the cavities. The characteristics of the accelerating structures of the TOROS and POLISCAN-3 systems are given in Table III.

\section{B. Features of the accelerating structure \\ 1. Dispersion characteristics}

The high-intensity electron beam changes resonant frequencies of cavities and can change the distribution of the electromagnetic field along the structure. To ensure good stability of the electromagnetic field, the following conditions are imposed: the coupling factor is sufficiently large, the dispersion characteristic is symmetrical, and resonant frequencies of the cavities are carefully adjusted.

In the accelerating structures of TOROS and POLISCAN-3 systems, the passband is about $90 \mathrm{MHz}$. In the course of manufacturing the cavities, they were adjusted with an accuracy of about $\pm 1 \mathrm{MHz}$. In the course of brazing the resonator, resonant frequencies of the cavities changed up to $5 \mathrm{MHz}$. After the brazing, cavities were adjusted with an accuracy of $\pm 1 \mathrm{MHz}$ by bending their walls. As a result, the accelerating field distribution is slightly affected by the beam loading and manufacturing errors.

\section{Accelerating field}

The length and number of cavities in the accelerating structure are selected as a trade-off of operation reliability against dimensions and weight of the radiation shielding. An acceleration rate is restricted by the danger of highvoltage discharges in the cavities. Figure 5 shows the measured distribution of electric field in the cavities of the TOROS system.

In the beginning of the structure, a large part of the injected beam is incident on the walls of the cavities and may produce emissions of secondary particles. In the first cavities, the intensity of the electric field is reduced, thus decreasing the probability of high-voltage discharges. The required field distribution is obtained by selection of coupling factors between the bunching and coupling cavities.

\section{Vacuum pumping and cooling the resonator}

To provide reliable operation at high field intensities, it is necessary to have high vacuum in the cavities and good vacuum conductivity of the resonator. The resonator shown in Fig. 3 is made from half cells. Figure 6 shows two half cells, each of them having two coupling slots. In order to minimize the direct coupling between cavities and improve symmetry of the dispersion characteristic, the slots in

TABLE III. Characteristics of accelerating structures.

\begin{tabular}{lcc}
\hline \hline System & TOROS & POLISCAN-3 \\
\hline Number of main cavities in bunching section & 3 & 3 \\
Number of main cavities in accelerating section & 8 & 14 \\
Maximum diameter of aperture $(\mathrm{mm})$ & 7 & 7 \\
Effective shunt impedance $(\mathrm{MOhm} / \mathrm{m})$ & 73 & 70 \\
Coupling factor & 0.03 & 0.03 \\
Maximum surface field $(\mathrm{MV} / \mathrm{m})$ & 35 & 32 \\
rf power loss in resonator $(\mathrm{kW})$ & 1.5 & 1.2 \\
Cooling water flow $(\mathrm{l} / \mathrm{min})$ & 5 & 5 \\
Vacuum $(\mathrm{Pa})$ & $10^{-5}$ & $10^{-5}$ \\
\hline \hline
\end{tabular}




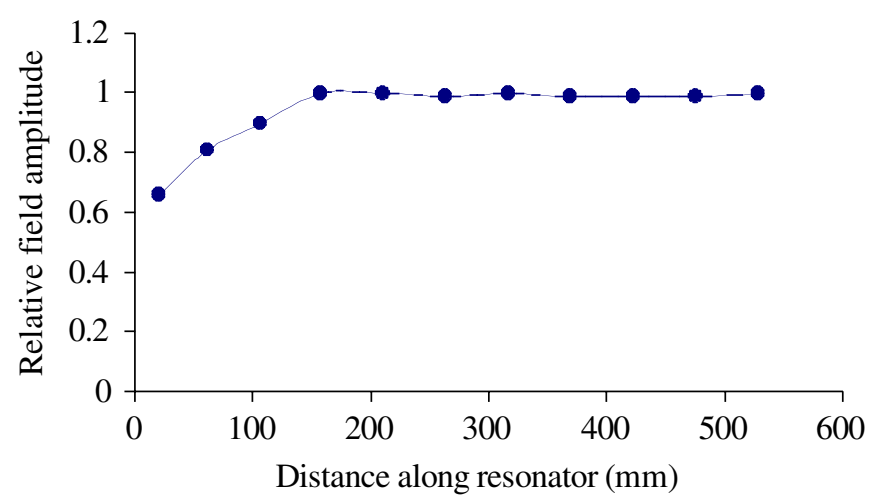

FIG. 5. (Color) Distribution of electric field in the resonator of the TOROS system.

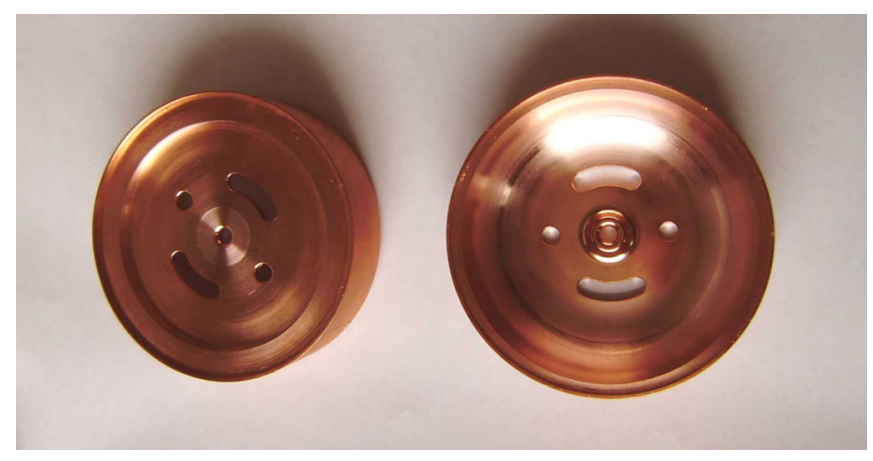

FIG. 6. (Color) Half cells of the accelerating structure.

adjacent walls are displaced by $90^{\circ}$ in azimuth. Since the coupling cavities are very thin, this displacement impairs the vacuum conductivity. Two additional holes are made in each wall that improve the vacuum conductivity but give only a slight direct coupling between the cavities.

It is important to select a proper scheme for cooling the resonator and maintaining its resonant frequency. The resonators of the TOROS and POLISCAN-3 systems are completely placed inside the water jackets. Water flows within the jacket symmetrically from its center to the ends. This design ensures efficient heat removal and good uniformity of resonant frequencies of the cavities. Temperature of the input water is stabilized. When full $\mathrm{rf}$ power is turned on, the mean temperature of the resonator is changed by about $2^{\circ} \mathrm{C}$ and resonant frequency of the resonator is changed by about $0.1 \mathrm{MHz}$, which is much less than the bandwidth of the pulling frequency of the magnetron.

\section{RADIO-FREQUENCY POWER SUPPLY}

\section{A. Characteristics of the rf power supply}

Basically, the scheme of the rf power supply is similar to a typical scheme of $\mathrm{rf}$ generators used for small linear accelerators [17]. However, the rf power supply for the
TABLE IV. Main characteristics of the rf power supply.

Frequency, regulated

Typical peak voltage

$2797 \pm 5 \mathrm{MHz}$

Peak current

$48 \mathrm{kV}$

Peak rf power at resonator input

$90 \mathrm{~A}$

Cross section of waveguide

$2.2 \mathrm{MW}$

Full length of waveguide

$(72 \times 34) \mathrm{mm}^{2}$

$1.8 \mathrm{~m}$

compact high-intensity accelerator has some particular features.

The rf generator is built around a MI-456A tunable magnetron. The main characteristics of the magnetron and waveguide are given in Table IV.

The Y-junction ferrite circulator is applied to ensure a matched load for the magnetron and protect the magnetron from damage or unstable operation. Since the magnetron has low stability of its own frequency, it operates in a "lock-on" regime, with the operating frequency being stabilized by the resonator. The matched load is placed in the third port of the circulator and has a regulated reflector ahead of it, which has a reflection factor of about 0.2. The intentionally introduced reflected signal at a certain phase pulls the magnetron frequency towards the resonant frequency of the accelerating resonator. The bandwidth of the pulling frequency is an important parameter defining stability of the accelerator operation. The bandwidth is dependent on a choice of rf generator parameters.

\section{B. Features of the rf power supply \\ 1. Adjustment of the waveguide section}

A full circuit of self-excitation of the rf generator includes the magnetron, accelerating resonator, and waveguide section. Of great importance is the phase of the reflected wave coming into the magnetron.

The calculations show that the magnetron operates in a lock-on regime if the phase of the reflected wave is in the range about $170^{\circ}$, that is about half of the full range of $360^{\circ}$. A maximum coefficient of frequency stabilization is in the center of this range. So the electric length of the waveguide section should be carefully adjusted. The adjustment may be executed with the use of a variable phase shifter located in the third port of the ferrite circulator.

\section{Coupling of the waveguide and accelerating resonator}

The operation of the rf generator in pulse mode has some specific features. To ensure stable operation of the generator, it is advantageous to adjust the coupling of the waveguide and accelerating resonator with overcoupling.

Figure 7 shows dependence of the bandwidth of the pulling frequency on the beam current in the accelerating resonator of the TOROS system. The dependencies are given for two cases. In the first case, the coupling of the waveguide and resonator is adjusted so that the complete 


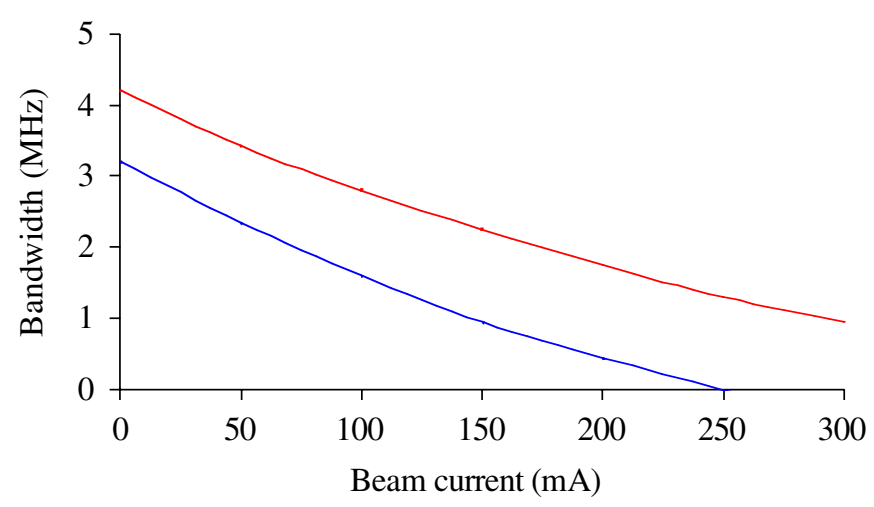

FIG. 7. (Color) Bandwidth of pulling frequency in relation to beam current: blue curve-matching at $250 \mathrm{~mA}$; red curveovercoupling.

matching takes place at a nominal peak current of $250 \mathrm{~mA}$. In the second case, the waveguide and resonator are overcoupled, with a reflection factor of 0.2 at the nominal current.

In a pulse accelerator, the beam current is varying on the edges of each pulse from zero to maximum. If the waveguide and resonator are matched at the nominal beam current, any instabilities and fluctuations may occur near the pulse top. If the waveguide and resonator are overcoupled, a sufficient value of the bandwidth of the pulling frequency is ensured throughout the whole pulse.

\section{Voltage stability}

Since magnetron frequency depends heavily on its anode voltage, the feeding pulse modulator should provide good stability of the output voltage. A variation of the modulator voltage by $0.1 \%$ may cause a change of the accelerating field intensity and electron energy of approximately $1 \%$. The shapes of some pulses measured in the accelerator of the TOROS system are shown in Fig. 8.

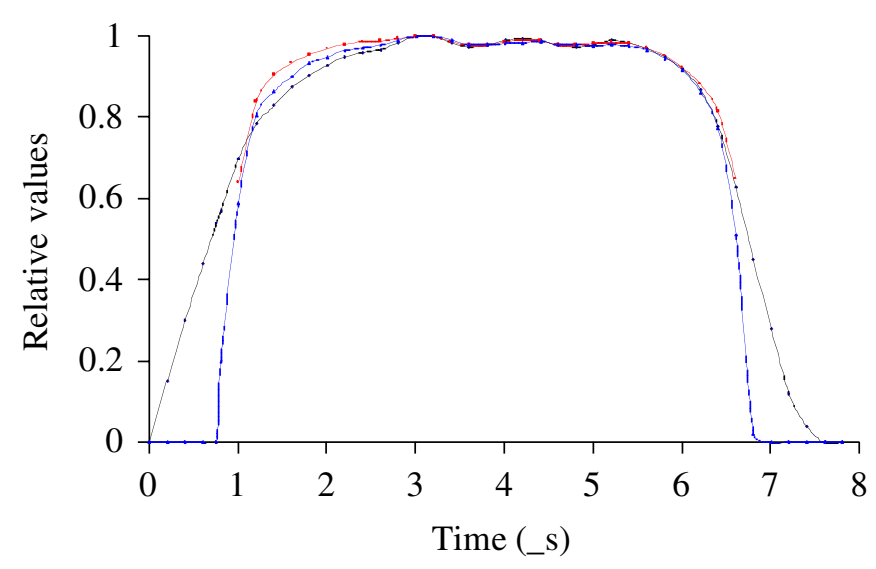

FIG. 8. (Color) Shape of pulses: black-anode current of the magnetron; blue-current of accelerated electrons; red-energy of accelerated electrons.
Capture of the injected beam in the acceleration mode begins at a magnetron current close to $50 \%$ of the nominal value. The energy of the accelerated electrons is rather high, even at the pulse edge, at the point where the magnetron current is $20 \%$ less than the nominal value. This provides good stabilization of magnetron frequency due to the frequency pulling effect.

\section{CONCLUSIONS}

Obtaining a high intensity of the electron beam in a compact accelerator with beam focusing by a rf field requires careful optimization of the accelerator and its components. The optimization process includes a choice of proper schemes and optimization of the beam dynamics and characteristics of the electron injector, accelerating structure, and rf power supply. In addition, it is useful to apply specific measures: a change of the channel aperture expanding progressively along the accelerator; a special design of the cathode, which generates the electron beam with azimuth components of electron velocities; a special distribution of the accelerating field with rising intensity along the accelerator; overcoupling of the waveguide and accelerating resonator.

The methods described were introduced in self-shielded compact electron accelerators designed for radiation sterilization and $\mathrm{x}$-ray cargo inspection. The accelerators have a peak beam current of up to $270 \mathrm{~mA}$ and demonstrate good performance.

[1] K. Irie, Y. Minowa, and S. Sawada, Jpn. J. Appl. Phys. 12, 277 (1973).

[2] V. M. Belugin, A. V. Mischenko, V. M. Pirozhenko, N. E. Rozanov, A. A. Zavadtsev, A. N. Korolev, and K. G. Simonov, Proceedings of the PAC-2001 (IEEE, Chicago, IL, 2001), pp. 2515-2517.

[3] V. M. Pirozhenko, V. M. Belugin, A. V. Mischenko, N. E. Rozanov, B.S. Sychev, V. V. Vetrov, Yu. Ya. Kokorovets, V.D. Ryzhikov, N. A. Shumeiko, S. Ya. Yatsenko, A. N. Korolev, K. G. Simonov, and V. V. Elyan, Proceedings of the EPAC-2006 (EPS-AG, Edinburgh, Scotland, 2006), pp. 2388-2390.

[4] L. Auditore, R. C. Barna, D. De Pasquale, A. Italiano, A. Trifiro, and M. Trimarchi, Phys. Rev. ST Accel. Beams 7, 030101 (2004).

[5] Y.Z. Lin, Proceedings of the PAC-2001 (Ref. [2]), pp. 2808-2810.

[6] H. Chen, Z. Xu, X. Jin, M. Li, X. Yang, H. Lu, L. Shan, X. Shen, Q. Pan, H. Hu, Y. Chen, Y. Wang, W. Li, H. Yu, Y. Chen, J. Liu, X. Liu, Z. Zhang, R. Deng, Q. Yu, H. Wang, and W. Bo, Proceedings of the APAC-2004 (Pohang Accelerator Laboratory, Gyeongju, Korea, 2004), pp. 429431.

[7] Q. Jin, D. Tong, Y. Lin, X. Sun, X. Tao, J. Sun, X. Duan, B. Chen, B. Sun, Y. Zou, and Y. Li, Proceedings of the LINAC-2004 (Gesellschaft für Schwerionenforschung, Lübeck, Germany, 2004), pp. 96-98. 
[8] S. H. Kim, H. R. Yang, S. I. Moon, J. Jang, Y. M. Gil, M. Cho, and W. Namkung, Proceedings of the PAC-2007 (IEEE, Albuquerque, New Mexico, USA, 2007), pp. 2823-2825.

[9] A. N. Korolev, A. V. Mischenko, V. M. Pirozhenko, K. G. Simonov, and A.I. Shapovalov, Biomedical Engineering (Radio Engineering, Moscow, Russia, 2000), pp. 5-16.

[10] V. M. Belugin, V. V. Vetrov, V. V. Elyan, A. V. Mischenko, V. M. Pirozhenko, N. E. Rozanov, V. M. Sitnikov, B. S. Sychev, A. N. Korolev, K. G. Simonov, Yu. Ya. Kokorovets, V.M. Svish, N. A. Shumeiko, S. Ya. Yatsenko, and V.D. Ryzhikov, Electronic Engineering (Istok, Moscow, Russia, 2007), pp. 11-19.

[11] E. Knapp, B. Knapp, and J. Potter, Rev. Sci. Instrum. 39, 979 (1968).

[12] V. G. Andreev, V. M. Belugin, V. G. Kulman, E. A.
Mirochnik, and V.M. Pirozhenko, Proceedings of the LINAC-72 (Los Alamos, New Mexico, 1972), pp. 114118.

[13] J. P. Labrie and J. McKeown, Nucl. Instrum. Methods 193, 437 (1982).

[14] N.E. Rozanov, Proceedings of Moscow Engineering Physics Institute MEPhI-2003 (MEPhI, Moscow, Russia, 2003), pp. 167-168.

[15] N.E. Rozanov, Proceedings of Moscow Engineering Physics Institute MEPhI-2005 (Ref. [14]), pp. 214-215.

[16] V. M. Belugin, V. M. Pirozhenko, N. E. Rozanov, and K. G. Simonov, X-ray Source (Russian Patent No. 2245588, 2003).

[17] C. J. Karzmark, C.S. Nunan, and E. Tanabe, Medical Electron Accelerators (McGraw-Hill, New York, 1993). 